\title{
Contents
}

1 Vibrational and Electronic Excitation of Molecules by Short-Pulse Strong Laser Fields

George N. Gibson, Li Fang, and Bradley Moser.................. 1

1.1 Introduction ................................. 1

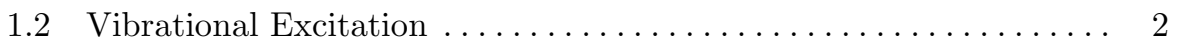

1.2.1 Vibrational Excitation Through Ionization............ 3

1.2.2 Vibrational Excitation Through $R$-Dependent Depletion.... 5

1.2.3 Vibrational Excitation Through Bond-Softening ......... 7

1.3 Electronic Excitation ........................... 9

1.3.1 Ionization to Charge-Asymmetric Dissociation Curves ...... 9

1.3.2 Resonant High-Order Multiphoton Excitation .......... 9

1.3 .3 Inner Orbital Ionization . . . . . . . . . . . . . . . . . . 15

1.3.4 Excitation Through Recollision.................. 16

1.4 Applications ................................ 17

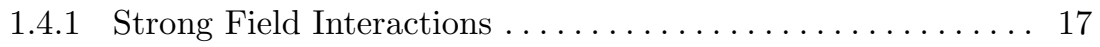

1.4.2 Quantum Tomography ...................... 17

1.4.3 Molecular Spectroscopy ....................... 18

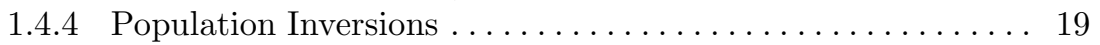

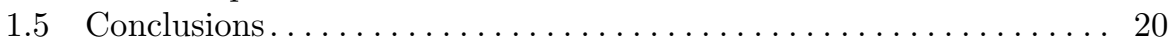

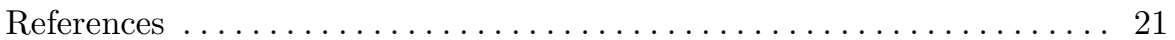

2 Coherent Lattice Oscillations in Solids and Their Optical Control Part I. Fundamentals and Optical Detection Techniques

Kunie Ishioka and Oleg V. Misochko ...................... 23

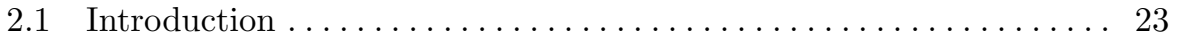

2.2 Generation of Coherent Phonons .................... 25

2.2.1 Impulsive Stimulated Raman Scattering ............ 25

2.2.2 Photocarrier-Mediated Excitation of Coherent Phonons . . . 27

2.3 Optical Detection of Coherent Phonons ................... 29

2.4 Electron-Phonon Coupling in Group V Semimetals ............ 30 
2.5 Coherent Phonons in Group IV Crystals and Graphitic Materials ...................... 33

2.5.1 Coherent Phonons in Tetrahedrally Bonded Crystals . . . . . . 33

2.5.2 Ultrafast Electron-Phonon Decoupling in Graphite . . . . . . . 35

2.5.3 Exciton-Phonon and Phonon-Phonon Couplings in Carbon Nanotubes ... . . . . . . . . . . . . . . . 37

2.6 Coherent Optical Phonons in Metals . . . . . . . . . . . . . . . . 38

2.7 Coherent Phonons in Other Materials ................... 41

2.8 Concluding Remarks . . . . . . . . . . . . . . . . . . . . . . . . . . . . . 42

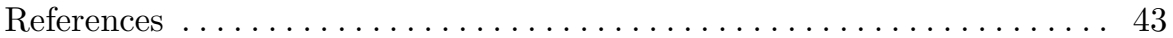

3 Coherent Lattice Oscillations in Solids and Their Optical Control Part II. New Detection Techniques and Optical Control

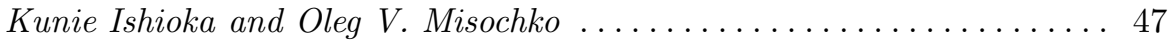

3.1 Coherent Phonons Detected by Novel Techniques . . . . . . . . . . . . 47

3.1.1 Time Resolved X-Ray Diffraction . . . . . . . . . . . . . . 47

3.1 .2 X-Ray Abrotption Spectroscopy . . . . . . . . . . . . . . . . . 50

3.1.3 Time-Resolved $\mathrm{THz}$ Spectroscopy ................ 50

3.1.4 Time-Resolved Photo-Emission Spectroscopy ... . . . . . . . 52

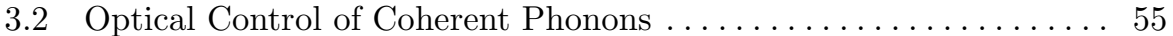

3.2.1 Optical Control at Low Excitation Density . . . . . . . . . . 55

3.2.2 Optical Control Near the Lindemann Limit . . . . . . . . . . . . . 58

3.2.3 Optical Control in Strongly Correlated Systems . . . . . . . . . 60

3.3 Concluding Remarks . . . . . . . . . . . . . . . . . . . . . . 60

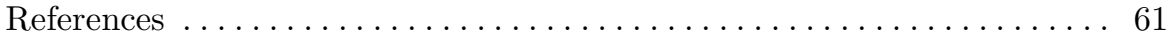

4 Heterodyne Interferometry Using High-Order Harmonic Generation in Mixed Gases

Tsuneto Kanai, Eiji J. Takahashi, Yasuo Nabekawa, and Katsumi Midorikawa ................................ 65

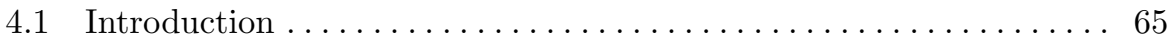

4.2 Theory of HHG in Mixed Gases and Heterodyne Interferometry for Detection of Ultrafast Molecular Dynamics . . . . . . . . . . . . 67

4.3 Destructive Interference During HHG in Mixed Gases . . . . . . . . . . 69 4.3 .1 Experimental . . . . . . . . . . . . . . . . . . . . 69

4.4 Application of the Heterodyne Interferometry to Attosecond Physics. . . . . . . . . . . . . . . . . . 72

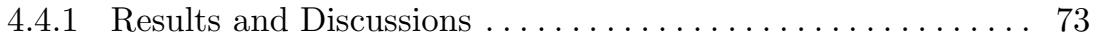

4.4 .2 Physical Origin of the Interference Signal . . . . . . . . . . . . 75

4.5 Conclusion . . . . . . . . . . . . . . . . . . . . . . . . . . . . 79

References ............................... 79 


\section{Propagation of Ultrashort Pulses in Condensed Media}

Aditya K. Dharmadhikari and Deepak Mathur ................. 81

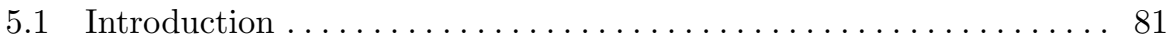

5.2 Propagation Effects: Filamentation $\ldots \ldots \ldots \ldots \ldots \ldots \ldots \ldots \ldots \ldots$

5.2 .1 Visualization of Filamentation $\ldots \ldots \ldots \ldots \ldots \ldots \ldots \ldots \ldots$

5.2 .2 Control Over the Onset of Filamentation . . . . . . . . . 86

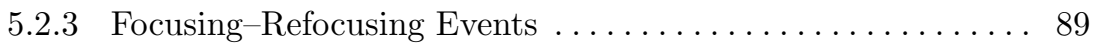

5.2.4 Other Control Issues: Multi-Filamentation . . . . . . . . . . . . . . . 91

5.3 Propagation Effects: Supercontinuum Generation .............. 94

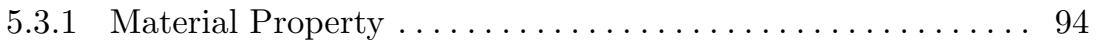

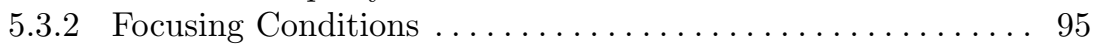

5.3 .3 Pulse Duration Dependence..................... 97

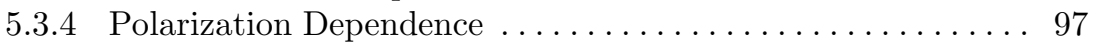

5.3 .5 Coherence ........................... 99

5.3 .6 Incident Power Dependence . . . . . . . . . . . . . . . . 99

5.4 Applications of White Light Generation and Filamentation . . . . . . 101

5.4.1 Supercontinuum Generation in Bio-Media . . . . . . . . . . . . 102

5.4 .2 Material Modification . . . . . . . . . . . . . . . . . . . . . . . . 103

References ..................................... 104

\section{On Lightning Control Using Lasers}

Jérôme Kasparian and Jean-Pierre Wolf . . . . . . . . . . . . . . . . 109

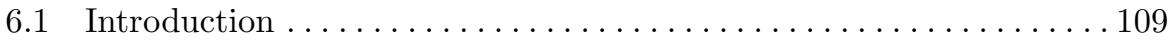

6.2 The Lightning Strike . . . . . . . . . . . . . . . . . . . . 110

6.3 Attempts to Trigger Lightning Using High-Energy Lasers . . . . . . . . 111

6.4 Control of High-Voltage Discharges Using Ultrashort Lasers . . . . . 112

6.5 Field Experiments Using Femtosecond Laser Filamentation . . . . . . . 114

6.6 Optimization of the Filament Effect

in Thunderstorms . . . . . . . . . . . . . . . . . 115

6.6.1 Optimization of the Plasma Density and Lifetime....... . 115

6.6.2 Mechanism of the Laser Filament Action

in Thunderclouds... . . . . . . . . . . . . . . . . . . . 115

6.6.3 Influence of the Geometric Configuration . . . . . . . . . . 117

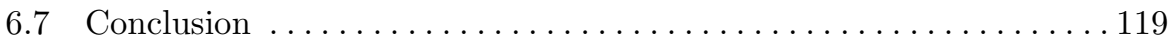

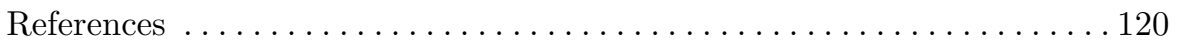

7 Advances in X-Ray Studies of Ultraintense Laser-Plasma Interactions

Leonida A. Gizzi................................ 123

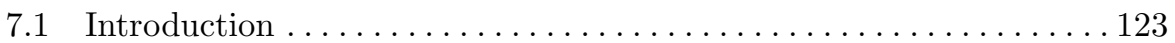

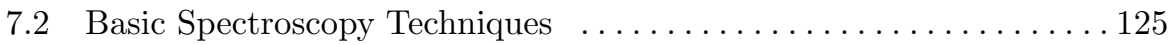

7.3 The Single Photon Detection Technique . . . . . . . . . . . . 127 


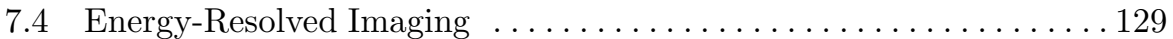

7.4.1 X-Ray Imaging of Interactions With Ti Foil Targets . . . . . . 130

7.4.2 Fast Electron Transport in Multilayer Targets............ 131

7.5 Summary and Conclusions .......................... 136

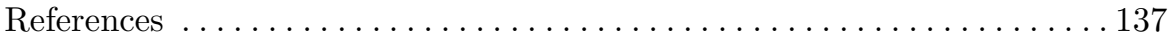

8 High Field Photonics in Laser Plasmas: Propagation Studies, Electron Acceleration, and Nuclear Activation With Ultrashort Intense Laser Pulses

Antonio Giulietti and Andrea Gamucci ....................... 139

$8.1 \quad$ Introduction . . . . . . . . . . . . . . . . . . . . . . . . 139

8.2 Studies on Laser Pulse Propagation ..................... 141

8.2.1 Propagation in Overdense Plasmas . ................... 141

8.2.2 Propagation in Underdense Plasmas and Pre-pulse Action ......................... 143

8.2.3 Preformed Pulse-Guiding Channels in Plasmas ........... 146

8.3 Electron Acceleration: Experiments and Simulations ............. 149

8.3.1 Laser Wakefield Acceleration and Associated Regimes ...... 149

8.3.2 Advanced Techniques and Record Results ............ 151

8.3.3 An Efficient Source of Relativistic Electrons

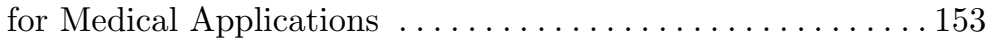

8.4 Nuclear Activation Using Electron Bunches from Laser Plasmas . . . 155

8.4.1 Basics of Nuclear Photo-Activation . . . . . . . . . . . . . 156

8.4.2 Activation Induced by Laser-Plasma Electrons ........... 157

8.4.3 An Example of High-Efficiency Photonuclear Activation in a Gas-Jet Experiment . . ....................... 159

8.4.4 Perspectives of Possible Applications . . . . . . . . . . . . . 160

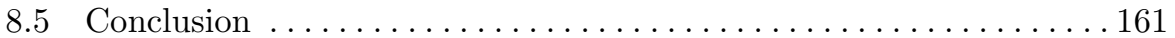

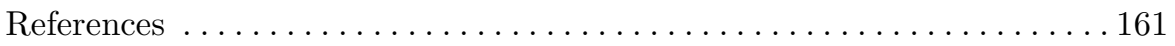

9 Laser Plasma Acceleration and Related Electromagnetic Sources

Danilo Giulietti and Luca Labate............................. 165

9.1 Introduction ................................... 165

9.2 Relativistic Electrons Sources . . . . . . . . . . . . . . . . . 169

9.3 Protons Sources ................................ 173

9.4 Laser Plasma Based e.m. Sources ........................ 176

9.5 Conclusions .................................. 180

References .......................................... 181

10 Laser-Driven Ion Generation with Short, Intense, and High Contrast Pulses

Tiberio Ceccotti, Anna Lévy, and Philippe Martin................. 187

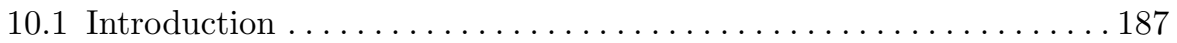

10.2 The TNSA Acceleration Mechanism: The Role of a Plasma Gradient . ......................... 188 
10.3 Ultra High Contrast Pulses with a Double Plasma Mirror . . . . . . 194

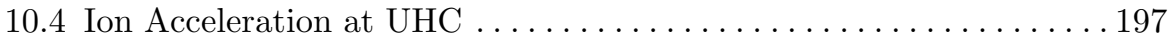

10.4.1 Acceleration Symmetry . . . . . . . . . . . . . . . . . 197

10.4.2 Laser Energy Transfer . . . . . . . . . . . . . . . . . . . . 201

10.5 An Analytical Model for Ion and Proton Emission at UHC . . . . . 202

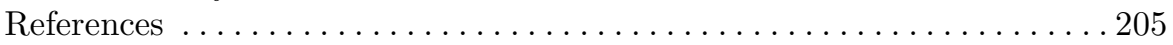

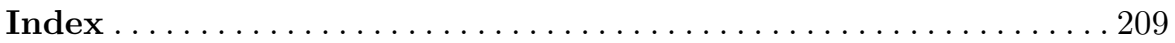

